\title{
Continuous rational maps into the unit 2-sphere
}

\author{
WOJCIECH Kucharz
}

\begin{abstract}
Investigated are continuous rational maps from a compact nonsingular real algebraic set into unit spheres. Special attention is devoted to such maps with values in the unit 2 -sphere.
\end{abstract}

Mathematics Subject Classification (2010) • 14P05, 14P25.

Keywords. Real algebraic set, Regular map, Continuous rational map, Approximation.

1. Introduction. Let $X$ and $Y$ be nonsingular real algebraic sets. A map $f: X \rightarrow Y$ is said to be continuous rational if it is continuous and there exists a regular map $\varphi: U \rightarrow Y$, defined on a Zariski open and dense subset $U$ of $X$, with $\left.f\right|_{U}=\varphi$. Let $P(f)$ denote the smallest algebraic subset of $X$ for which the restriction map $\left.f\right|_{X \backslash P(f)}: X \backslash P(f) \rightarrow Y$ is regular. Thus $P(f)$ is the indeterminacy locus of the rational map from $X$ into $Y$ determined by $\varphi$. Maps with $f(P(f)) \neq Y$ will be called nice. There exist continuous rational maps which are not nice, cf. [6, Example 2.2]. Continuous rational maps form a natural intermediate class between regular and continuous semi-algebraic maps, with many specific properties, cf. [3,5-7].

In $[6,7]$, continuous rational maps with values in the unit $p$-sphere

$$
\mathbb{S}^{p}=\left\{\left(u_{1}, \ldots, u_{p+1}\right) \in \mathbb{R}^{p+1} \mid u_{1}^{2}+\cdots+u_{p+1}^{2}=1\right\}
$$

are investigated. They behave like regular maps in problems involving homotopy or approximation, provided that $p=1$, cf. [6, Corollary 1.4]. However, one encounters new phenomena for $p \geq 2$, cf. [6, p. 518] and [7, Example 1.8]. The main results of the present paper, Theorems 1.2 and 1.4, concern approximation of continuous maps from $X$ into $\mathbb{S}^{2}$ by continuous rational maps. Here approximation refers to the compact open topology.

The author was partially supported by NCN grant 2011/01/B/ST1/01289. 
First some preparation is necessary. Let $M$ be a smooth (of class $\mathcal{C}^{\infty}$ ) manifold, and let $N$ be a smooth submanifold of $M$ of codimension $p$. By convention, submanifolds are assumed to be closed subsets of the ambient manifold. Assume that the normal bundle of $N$ in $M$ is, oriented, and denote by $\tau_{N}^{M}$ the Thom class of $N$ in the cohomology group $H^{p}(M, M \backslash N$; $\mathbb{Z})$, cf. [8, p. 118]. The image of $\tau_{N}^{M}$ by the restriction homomorphism $H^{p}(M, M \backslash N ; \mathbb{Z}) \rightarrow H^{p}$ $(M ; \mathbb{Z})$, induced by the inclusion map $M \hookrightarrow(M, M \backslash N)$, will be denoted by $[N]^{M}$ and called the cohomology class represented by $N$. If $M$ is oriented and $N$ is endowed with the compatible orientation, then $[N]^{M}$ is up to sign Poincaré dual to the homology class in $H_{*}(M ; \mathbb{Z})$ represented by $N$, cf. [8, p. 136].

Let $P$ be a smooth manifold, and let $Q$ be a smooth submanifold of $P$. A continuous map $f: M \rightarrow P$ is said to be transverse to $Q$ if it is smooth in an open neighborhood $U$ of $f^{-1}(Q)$ in $M$ and the restriction map $\left.f\right|_{U}: U \rightarrow P$ is transverse to $Q$ in the usual sense. In that case, if the normal bundle of $Q$ in $P$ is oriented and the normal bundle of the smooth submanifold $N:=$ $f^{-1}(Q)$ of $M$ is endowed with the orientation induced by $f$, then $\tau_{N}^{M}=f^{*}\left(\tau_{Q}^{P}\right)$, where $f$ is regarded as a map from $(M, M \backslash N)$ into $(P, P \backslash Q)$ (this follows from $[4$, p. 117 , Theorem 6.7$])$. In particular, $[N]^{M}=f^{*}\left([Q]^{P}\right)$.

The unit sphere $\mathbb{S}^{p}$ is considered with a fixed orientation. Hence any point $z$ in $\mathbb{S}^{p}$ can be regarded as a smooth submanifold of $\mathbb{S}^{p}$ with oriented trivial normal bundle. Let $\sigma_{p}$ denote the generator of the cohomology group $H^{p}\left(\mathbb{S}^{p} ; \mathbb{Z}\right) \cong \mathbb{Z}$ determined by the orientation of $\mathbb{S}^{p}$. In other words, $\sigma_{p}=[z]^{\mathbb{S}^{p}}$.

Assumption. In the rest of this section, the algebraic set $X$ is assumed to be compact.

A cohomology class $u$ in $H^{p}(X ; \mathbb{Z})$ is said to be adapted if there exists an algebraic codimension $p$ subset $V$ of $X$ such that $\operatorname{Reg}(V)$ is a compact smooth submanifold of $X$ with trivial normal bundle and $u=[\operatorname{Reg}(V)]^{X}$ when the normal bundle of $\operatorname{Reg}(V)$ is suitably oriented. Here $\operatorname{Reg}(V)$ stands for the set of nonsingular points of $V$.

Proposition 1.1. If $f: X \rightarrow \mathbb{S}^{p}$ is a nice continuous rational map, then the cohomology class $f^{*}\left(\sigma_{p}\right)$ in $H^{p}(X ; \mathbb{Z})$ is adapted.

Proof. Since $f(P(f))$ is a proper compact subset of $\mathbb{S}^{p}$, it follows from Sard's theorem that the regular map $\left.f\right|_{X \backslash P(f)}: X \backslash P(f) \rightarrow \mathbb{S}^{p}$ is transverse to some point $z$ in $\mathbb{S}^{p} \backslash f(P(f))$. Hence $N:=f^{-1}(z)$ is a nonsingular Zariski closed subset of $X \backslash P(f)$. If $V$ is the Zariski closure of $N$ in $X$, then $V \backslash N$ is a Zariski closed subset of $X$ contained in $P(f)$, with $\operatorname{dim}(V \backslash N)<\operatorname{dim} V$. Thus $N=\operatorname{Reg}(V)$, the set $N$ being compact. Since the continuous map $f: X \rightarrow \mathbb{S}^{p}$ is transverse to $z$, one has $f^{*}\left(\sigma_{p}\right)=[\operatorname{Reg}(V)]^{X}$, provided that $\operatorname{Reg}(V)$ is endowed with the orientation induced by $\left.f\right|_{X \backslash P(f)}$.

Denote by $A^{p}(X ; \mathbb{Z})$ the subgroup of $H^{p}(X ; \mathbb{Z})$ generated by all adapted cohomology classes. It is an open problem whether or not for a continuous rational map $f: X \rightarrow \mathbb{S}^{p}$, the cohomology class $f^{*}\left(\sigma_{p}\right)$ is in $A^{p}(X ; \mathbb{Z})$. This problem is of particular interest for $p=2$ in view of the following result. 
Theorem 1.2. If $h: X \rightarrow \mathbb{S}^{2}$ is a continuous map such that the cohomology class $h^{*}\left(\sigma_{2}\right)$ is in $A^{2}(X ; \mathbb{Z})$, then $h$ can be approximated by continuous rational maps.

The proof is postponed until Sect. 2 .

Corollary 1.3. Let $C_{1}, \ldots, C_{n}$ be compact connected nonsingular real algebraic sets of dimension 1. Then any continuous map from $C_{1} \times \cdots \times C_{n}$ into $\mathbb{S}^{2}$ can be approximated by continuous rational maps.

Proof. It suffices to observe that

$$
A^{2}\left(C_{1} \times \cdots \times C_{n} ; \mathbb{Z}\right)=H^{2}\left(C_{1} \times \cdots C_{n} ; \mathbb{Z}\right)
$$

and apply Theorem 1.2.

Denote by $A^{p}(X ; \mathbb{Z} / 2)$ the image of $A^{p}(X ; \mathbb{Z})$ by the reduction modulo 2 homomorphism

$$
H^{p}(X ; \mathbb{Z}) \rightarrow H^{p}(X ; \mathbb{Z} / 2) .
$$

The reduction modulo 2 of $\sigma_{p}$, denoted by $\bar{\sigma}_{p}$, is a generator of the cohomology group $H^{p}\left(\mathbb{S}^{p} ; \mathbb{Z} / 2\right) \cong \mathbb{Z} / 2$.

Theorem 1.4. Assume that the cohomology group $H^{2}(X ; \mathbb{Z})$ is generated by the cohomology classes of the form $[N]^{X}$, where $N$ is a codimension 2 compact smooth submanifold of $X$ with oriented trivial normal bundle. If $h: X \rightarrow \mathbb{S}^{2}$ is a continuous map such that the cohomology class $h^{*}\left(\bar{\sigma}_{2}\right)$ is in $A^{2}(X ; \mathbb{Z} / 2)$, then $h$ can be approximated by continuous rational maps.

The proof will be given in the next section. It is not clear whether Theorems 1.2 and 1.4 can be extended to maps with values in $\mathbb{S}^{p}$ for $p \geq 3$.

2. Proofs. For any topological space $T$, denote by $\varepsilon_{T}^{k}(\mathbb{C})$ the standard trivial $\mathbb{C}$-vector bundle on $T$ with total space $T \times \mathbb{C}^{k}$.

A topological $\mathbb{C}$-vector bundle $\xi$ on a compact nonsingular real algebraic set $X$ is said to admit a rational structure if there exist a topological $\mathbb{C}$-vector subbundle $\eta$ of $\varepsilon_{X}^{k}(\mathbb{C})$, for some $k$, and a Zariski open and dense subset $U$ of $X$ such that $\xi$ is isomorphic to $\eta$ and the restriction $\left.\eta\right|_{U}$ is an algebraic $\mathbb{C}$-vector subbundle of $\varepsilon_{U}^{k}(\mathbb{C})$, cf. [6, Definition 3.2].

Identify $\mathbb{S}^{2}$ with the complex projective line $\mathbb{P}^{1}(\mathbb{C})$, and denote by $\gamma_{2}$ the $\mathbb{C}$-line bundle on $\mathbb{S}^{2}$ corresponding to the universal $\mathbb{C}$-line bundle $\lambda$ on $\mathbb{P}^{1}(\mathbb{C})$. Explicitly, let $a=(0,1)$ be a point in $\mathbb{C} \times \mathbb{R}=\mathbb{R}^{3}$, and let $\rho: \mathbb{S}^{2} \backslash\{a\} \rightarrow \mathbb{C}$ be the stereographic projection. Then $\alpha: \mathbb{S}^{2} \rightarrow \mathbb{P}^{1}(\mathbb{C})$,

$$
\alpha(x)= \begin{cases}(\rho(x): 1) & \text { for } x \text { in } \mathbb{S}^{2} \backslash\{a\} \\ (1: 0) & \text { for } x=a,\end{cases}
$$

is a smooth diffeomorphism and $\gamma_{2}:=\alpha^{*} \lambda$. In particular, $\gamma_{2}$ is an algebraic $\mathbb{C}$-vector subbundle of $\varepsilon_{\mathbb{S}^{2}}^{2}(\mathbb{C})$. The first Chern class $c_{1}\left(\gamma_{2}\right)$ of $\gamma_{2}$ is a generator 
of the cohomology group $H^{2}\left(\mathbb{S}^{2} ; \mathbb{Z}\right)$. It can be assumed that $\mathbb{S}^{2}$ is oriented in such a way that $\sigma_{2}=c_{1}\left(\gamma_{2}\right)$.

Proof of Theorem 1.2. If $u$ is an adapted cohomology class in $H^{2}(X ; \mathbb{Z})$, then so is $-u$. Since the cohomology class $h^{*}\left(\sigma_{2}\right)$ is in $A^{2}(X ; \mathbb{Z})$, one can find nonnegative integers $k_{1}, \ldots, k_{r}$ and adapted cohomology classes $u_{1}, \ldots, u_{r}$ in $H^{2}(X ; \mathbb{Z})$ such that

$$
h^{*}\left(\sigma_{2}\right)=k_{1} u_{1}+\cdots+k_{r} u_{r} .
$$

According to [6, Theorem 2.5], for each integer $i$ satisfying $1 \leq i \leq r$, there exists a continuous rational map $f_{i}: X \rightarrow \mathbb{S}^{2}$ with $u_{i}=f_{i}^{*}\left(\sigma_{2}\right)$. The $\mathbb{C}$-line bundle $\xi_{i}:=f_{i}^{*} \gamma_{2}$ on $X$ admits a rational structure. Consequently, the $\mathbb{C}$-line bundle

$$
\xi:=\xi_{1}^{\otimes k_{1}} \otimes \cdots \otimes \xi_{r}^{\otimes k_{r}}
$$

on $X$ admits a rational structure, cf. [6, Proposition 3.3]. One has

$$
c_{1}\left(\xi_{i}\right)=f_{i}^{*}\left(c_{1}\left(\gamma_{2}\right)\right)=f_{i}^{*}\left(\sigma_{2}\right)=u_{i},
$$

and hence $c_{1}(\xi)=h^{*}\left(\sigma_{2}\right)$. Furthermore,

$$
h^{*}\left(\sigma_{2}\right)=h^{*}\left(c_{1}\left(\gamma_{2}\right)\right)=c_{1}\left(h^{*} \gamma_{2}\right) .
$$

Thus $c_{1}(\xi)=c_{1}\left(h^{*} \gamma_{2}\right)$, which implies that the $\mathbb{C}$-vector bundles $\xi$ and $h^{*} \gamma_{2}$ are isomorphic. It follows that $h^{*} \gamma_{2}$ admits a rational structure and hence according to [6, Corollary 3.8], the map $h$ can be approximated by continuous rational maps.

Proof of Theorem 1.4. Since the cohomology class $h^{*}\left(\bar{\sigma}_{2}\right)$ is in $A^{2}(X ; \mathbb{Z} / 2)$, by the universal coefficient theorem, the cohomology class $h^{*}\left(\sigma_{2}\right)$ can be expressed as $h^{*}\left(\sigma_{2}\right)=u+2 v$, where $u$ is in $A^{2}(X ; \mathbb{Z})$ and $v$ is in $H^{2}(X ; \mathbb{Z})$. By assumption, the cohomology class $v$ is a linear combination with integer coefficients of cohomology classes of the form $[N]^{X}$, where $N$ is a codimension 2 compact smooth submanifold of $X$ with oriented trivial normal bundle. According to Theorem 1.2, it suffices to prove that the cohomology class $2[N]^{X}$ is adapted.

Since the normal bundle of $N$ in $X$ is trivial, there exists an isotopic copy $N^{\prime}$ of $N$ such that $N \cap N^{\prime}=\varnothing$ and $N \cup N^{\prime}$ is the boundary of a compact smooth manifold with boundary, embedded in $X$ with trivial normal bundle. Hence $N \cup N^{\prime}$ can be represented as $N \cup N^{\prime}=f^{-1}(0)$, where $f: X \rightarrow \mathbb{R}^{2}$ is a smooth map transverse to 0 in $\mathbb{R}^{2}$ (cf. for example [2, Theorem 1.12]). By the Weierstrass approximation theorem, there exists a regular map $g: X \rightarrow \mathbb{R}^{2}$ arbitrarily close to $f$ in the $\mathcal{C}^{\infty}$ topology. If $g$ is sufficiently close to $f$, then $g$ is transverse to 0 and the smooth submanifold $N \cup N^{\prime}$ is isotopic in $X$ to the nonsingular algebraic subset $Z:=g^{-1}(0)$, cf. [1, p. 51]. By construction, $2[N]^{X}=[Z]^{X}$, provided that the normal bundle of $Z$ in $X$ is suitably oriented. Hence the cohomology class $2[N]^{X}$ is adapted, as required. 
Open Access. This article is distributed under the terms of the Creative Commons Attribution License which permits any use, distribution, and reproduction in any medium, provided the original author(s) and the source are credited.

\section{References}

[1] R. Abraham and J. Robbin, Transversal Mappings and Flows, New York, Benjamin, 1967.

[2] J. Bochnak and W. Kucharz, Complete intersections in differential topology and analytic geometry, Boll. Un. Mat. Ital. B 10 (1996), 1019-1041.

[3] G. Fichou, J. Huisman, F. Mangolte, And J.-Ph. Monnier, Fonctions régulues, arXiv:1112.3800 [math. AG].

[4] M. W. Hirsch, Differential Topology, Springer, 1997.

[5] J. Kollár and K. NowaK, Continuous rational maps on real and p-adic varieties, arXiv:1301.5048 [math. AG].

[6] W. Kucharz, Rational maps in real algebraic geometry, Adv. Geom. 9 (2009), $517-539$.

[7] W. Kucharz, Approximation by continuous rational maps into spheres, J. Eur. Math. Soc., to appear.

[8] J. W. Milnor and J. D. Stasheff, Characteristic Classes, Ann. Math. Studies 76, Princeton University Press, Princeton, NJ, 1974.

WOJCIECH KUCHARZ

Institute of Mathematics,

Faculty of Mathematics and Computer Science,

Jagiellonian University,

Łojasiewicza 6, 30-348 Kraków, Poland

e-mail: Wojciech.Kucharz@im.uj.edu.pl

Received: 26 July 2013 